\title{
DESAFÍOS CONTEMPORÁNEOS DEL ESTADO, LA DEMOCRACIA Y LA CIUDADANÍA
}

\author{
JUAN CARLOS BENÍTEZ LÓPEZ ${ }^{1}$ \\ ORLANDO JAVIER GALEANO VIDES ${ }^{2}$ \\ JENNY SOBEYDA SUAZO SEVILLA ${ }^{3}$ \\ LEONARDO EUCEDA AMAYA ${ }^{4}$
}

Doctorandos en Ciencias del Desarrollo Humano, Universidad Nacional Autónoma de Honduras

DOI: $10.5377 /$ rpdd.v6i1.12420

Recibido: agosto, 2021

Aceptado: septiembre, 2021

\section{AGRADECIMIENTO}

L

os integrantes del Círculo Temático Estado, Ciudadanía, Gobernabilidad, Descentralización y Derechos Humanos del Programa de Doctorado en Ciencias del Desarrollo Humano de la UNAH, deseamos agradecer profundamente al Doctor Juan José García Escribano, de la Universidad de Murcia, España, por todo el asesoramiento dado en el proceso de elaboración del presente artículo que hemos titulado: "Desafíos contemporáneos del Estado, la Democracia y la Ciudadanía". El apoyo dado y los aprendizajes recibidos han sido múltiples. Gracias Doctor García Escribano por mostrarnos siempre el camino y por aportarnos importantes sugerencias, bibliografías, comentarios, revisiones, todo lo cual nos facilitó la culminación de este importante ejercicio para nuestra formación. Nuestro agradecimiento al Doctor Pedro Morazán quién desde Berlín, Alemania, nos realizó una revisión exhaustiva, excelentes comentarios e importantes sugerencias, que sin duda ayudó a profundizar las reflexiones y a replantear algunos aspectos, ayudando con ello a una mejor presentación del artículo en su versión final. Muchas gracias.

\section{RESUMEN}

El Estado, la democracia y la ciudadanía son fundamentales para concretizar el desarrollo humano, ya que el buen o mal desempeño determinará la realización o no de la población. Por ello, en el presente artículo planteamos los distintos

1MSc. JUAN CARLOS BENÍTEZ LÓPEZ, Asesor en políticas públicas, benitezlopezjuancarlos@gmail.com, Orcid: https://orcid.org/ 0000-0003-0974-8659.

2MSc.ORLANDO JAVIER GALEANO VIDES, Docente Universidad Nacional Autónoma de Honduras CUROC, orlando.galeano@unah.edu.hn, Orcid: https://orcid.org/0000-0002-4957-4457.

3MSc. JENNY SOBEYDA SUAZO SEVILLA, Especialista en gestión académica superior UNAH, jenny. suazo@unah.edu.hn, Orcid: https://orcid.org/0000-0001-6434-0758.

4MSc. LEONARDO EUCEDA AMAYA, Docente Universidad Nacional Autónoma de Honduras, leoeucedaamaya1@yahoo.com, Orcid: https://orcid.org/0000-0001-5979-1300. 
debates contemporáneos que están en curso, resaltando los desafíos que habrán que afrontarse a fin de alcanzar un desarrollo humano sostenible, lo cual implica no solo una reconceptualización del Estado y una profundización de la democracia, sino que también implica la ampliación de los mecanismos en los cuales la ciudadanía se involucre en los asuntos públicos, lo cual proporcionará legitimidad al Estado, viabilidad a la democracia y nuevos marcos políticos de participación y desarrollo incluyente.

\title{
PALABRAS CLAVE
}

Estado, democracia, gobernabilidad y ciudadanía

\begin{abstract}
The State, democracy and citizenship are fundamental to achieve human development, since good or bad performance will determine the achievement or not of the population. Therefore, in this article we present the different contemporary debates that are underway, highlighting the challenges that will have to be faced in order to achieve sustainable human development. This implies not only a reconceptualization of the State and a deepening of democracy, but also the expansion of the mechanisms in which citizens are involved in public affairs, which will provide legitimacy to the State, viability of democracy and new political frameworks for participation and inclusive development.
\end{abstract}

\section{KEYWORDS}

State, democracy, governance and citizenship

\section{INTRODUCCIÓN}

El presente artículo corresponde al trabajo realizado por el Círculo Temático Estado, Ciudadanía, Gobernabilidad, Descentralización y Derechos Humanos del Programa del Doctorado de la UNAH. Como parte del proceso de formación en el programa del Doctorado, hemos sido partícipes del Foro "Debate Contemporáneo del Desarrollo Humano" realizado en el mes de mayo del año 2021, el cual contó con exponentes experimentados en cada uno de los círculos temáticos organizados, quienes proporcionaron aportes importantes en la temática correspondiente a nuestro circulo.

El objetivo del presente artículo es poner en perspectiva los distintos planteamientos teóricos que están en el debate con relación al Estado, la democracia y la ciudadanía, para con ello explicar la situación actual en la sociedad hondureña y 
plantear los desafíos que habrá que afrontar a fin de alcanzar el desarrollo humano en sus dimensiones: social, económica, política, cultural y ecológica.

Desde los marcos teóricos actualmente planteados, partimos en analizar qué es el Estado y el por qué se está debatiendo un nuevo papel en la conducción de las sociedades. No hay duda de que el Estado es fundamental, pero al ampliar el debate hacia una mayor democracia y mayor involucramiento de la ciudadanía en los asuntos públicos, nos encontramos con planteamientos de que el Estado debería ser reformado a fin de que responda a los nuevos desafíos que la sociedad contemporánea demanda.

\section{Hacia un nuevo papel del Estado}

Con la consolidación del Estado moderno ha prevalecido el pensamiento sobre la necesidad de responder sobre su quehacer y su función dentro de las sociedades. La existencia de una diversidad de escuelas de pensamiento implica igualmente que la concepción sobre el Estado ha sido diversa. El Estado tiene que ver con la política, con la sociedad, con el gobierno y con el poder. El Estado ha sido la forma dominante de organización del poder político. Al analizar las sociedades contemporáneas en relación con el Estado, la democracia y la ciudadanía, podemos concluir que vivimos un tiempo de incertidumbre, de desconfianza, de miedo, lo que el sociólogo Zygmunt Bauman (2008) llama "política y sociedad líquida" precisamente porque ya no es posible establecer relaciones duraderas. Es decir: «En esta nueva etapa, fase posterior a la anterior en la que todo era sólido, los conceptos se convierten en licuados; y los acuerdos son precarios, temporales, pasajeros; válidos solo hasta un nuevo aviso»

En un sentido parecido, Beck (2017), en su obra, La sociedad del riesgo global, plantea "la sociedad del riesgo" y llama la atención sobre la mundialización del riesgo, ya que los Estados pierden capacidad de garantizar los derechos de sus ciudadanos, generando una crisis política en la cual la democracia es menos valorada, las instituciones no funcionan, la falta de oportunidades genera conflictos y la desigualdad social se convierte en un tema político.

Beck señala que "los riesgos globales tienen efectos similares: intensifican en todo el mundo relaciones sociales que incluso en la antigua 'periferia' influyen en los acontecimientos que se producen en las 'antiguas metrópolis' y viceversa. Los riesgos globales, por tanto, ya no son procesos de imperialismo unidireccional, antes bien, son desordenados y caóticos. La extensión del riesgo, por muy irregular y esporádica que sea, ha dado lugar a la extensión de las incertidumbres prefabricadas o, dicho de otro modo, a la generación de las incertidumbres prefabricadas" (Beck, 2017, pp. 176-177). 
A partir de la década de los setenta se lleva gestando la configuración de una nueva realidad, la cual se ha visto acelerada por la crisis económico-financiera del año 2008 y la pandemia del COVID-19. Los cambios eminentes han sido relacionados con la emergencia de una nueva ciudadanía digital, la ruptura del contrato social, el fin de los grandes relatos, una reconceptualización de la sociedad actual que, dependiendo del autor o del planteamiento teórico, es calificada como sociedad de red, sociedad digital, sociedad fragmentada, sociedad líquida, sociedad global, sociedad postindustrial, sociedad de paradojas, entre muchas otras formas de denominarla.

Según Richard Sennet (2011), se ha pasado de un modelo social de convivencia a un modelo de sociedad fragmentada en la que se están perdiendo aceleradamente los significados y cada vez existe menos comunicación entre la diversidad social. Sennet (2011) plantea que "la sociedad que habitamos actualmente se encuentra agobiada por las consecuencias de esa historia, la destrucción de la res pública por la creencia de que los significados sociales son generados por los sentimientos de los seres humanos individuales. Este cambio ha oscurecido para nosotros dos áreas de la vida social. Una es el dominio del poder, la otra es el dominio del entorno en el que vivimos" (p. 415).

Conforme a lo anterior, Sennet (2011) plantea que el Estado contemporáneo está fragmentado debido a una sociedad que también es producto de fragmentaciones de muy diversa tipología: 1. Fragmentación Productiva (División del trabajo) 2. Fragmentación Social (Desigualdad) 3. Fragmentación Estructural (Complejidad) 4. Fragmentación Cultural (Individualismo).

Nuestras sociedades se encuentran frente a una serie de desafíos como la superación de la pobreza, el hambre, el cambio climático, las migraciones forzadas, entre otras, como consecuencia de las diversas crisis sociales, políticas, económicas, ambientales y financieras que se han sucedido en los últimos tiempos, tanto por la deficiencia en los tratamientos de los asuntos públicos por los propios actores gubernamentales, como por la aparición de nuevos actores y roles en el espacio público, como por ejemplo los movimientos de las mujeres, los pueblos originarios, los movimientos de la diversidad sexual, ambientalistas entre otros, con la pretensión de canalizar demandas ciudadanas no satisfechas y por la exigencia de abrir el gobierno para establecer un horizonte institucional y político menos complejo.

El Estado, siempre que sea de derecho y democrático, reconoce y garantiza esa participación ciudadana que fortalece y enriquece al Estado mismo. Nuestras sociedades siempre han requerido de una ciudadanía activa que participe en su 
construcción. Una ciudadanía activa nos acerca a una democracia ética y comprometida con la igualdad.

Para Boaventura de Sousa Santos (2005), el nuevo Estado debe ser un Estado experimental y en la experimentación continua con una activa participación de los ciudadanos estará la sostenibilidad del bienestar: "el Estado experimental debe por tanto asegurar no sólo la igualdad de oportunidades entre los distintos proyectos de institucionalidad democrática, sino - y este es el segundo principio de la experimentación política - unas pautas mínimas de inclusión que hagan posible una ciudadanía activa capaz de controlar, acompañar y evaluar la valía de los distintos proyectos. Estas pautas son necesarias para hacer de la inestabilidad institucional un ámbito de deliberación democrática" (p. 370).

\section{Estado y gobernabilidad}

La gobernabilidad es un concepto distinto al concepto de gobernanza. De acuerdo con Joan Prats (1996), al hablar de gobernabilidad democrática la concebimos "como la capacidad de un sistema social democrático para autogobernarse enfrentando positivamente los retos y oportunidades que tenga planteados" (p. 449).

Bobbio, Matteucci y Pasquino (2002) definen el término de gobernabilidad como "la relación de gobierno, es decir, la relación de gobernantes y gobernados" (p. 703), por lo que la relación compleja entre estos dos sujetos es lo que posibilita el poder hablar de gobernabilidad. Un sistema social sería gobernable cuando esté estructurado sociopolíticamente de tal modo que todos los actores estratégicos se interrelacionen para tomar decisiones colectivas y resolver sus conflictos, conforme a un sistema de reglas y de procedimientos formales o informales.

Según Prats (1996), "la gobernabilidad democrática no se refiere, pues, tanto a los atributos de un régimen democrático cuanto a las capacidades de una determinada sociedad democrática para enfrentar los retos y oportunidades específicos que tiene planteados" (p. 449). La gobernabilidad vendría a ser un atributo, una cualidad de algunas sociedades en las cuales los actores estratégicos, (los actores claves de esa sociedad) pueden interrelacionarse precisamente para producir decisiones, pero sobre la base de un sistema de reglas y de procedimientos relativamente estables y predecibles.

El mismo Prats (1996), siguiendo a Kooiman (1993), señala que "una estrategia de gobernabilidad democrática es, pues, una estrategia de construcción de capacidades. Tales capacidades dependen de la interrelación entre el sistema 
institucional existente (governance), de las capacidades de los actores políticos, económicos y sociales presentes (governing actors), y, finalmente, de la cantidad y calidad del liderazgo transformacional disponible" (p. 449).

De acuerdo con Prats (1996), "la gobernabilidad democrática presupone la existencia de democracia, pero ésta no es condición suficiente para que exista gobernabilidad democrática. La gobernabilidad democrática se dará sólo cuando la toma de decisiones de autoridad y la resolución de conflictos entre los actores estratégicos se produzca conforme a un sistema de reglas y fórmulas que podamos calificar como democracia.

No podemos avanzar, pues, en el concepto de gobernabilidad democrática, sin precisar el concepto de democracia” (p. 133). Por lo anterior, para lograr la legitimidad de la gobernabilidad no solo es responsabilidad del gobierno a través de sus diferentes instancias, sino de la interacción de actores como la empresa privada y la misma sociedad civil, y todas ellas deberán propiciar e impulsar políticas públicas que permitan la satisfacción de las necesidades más básicas de la población y el despegue del desarrollo a nivel de un territorio.

Independientemente del concepto o enfoque que se adopte, más allá que algunos autores presten más atención a un elemento que a otro, para Antonio Camou (2013) en su obra Gobernabilidad y Democracia, plantea que la gobernabilidad tiene tres componentes básicos:

i.la legitimidad que tiene que ver con el origen del gobierno y con la eficacia con la que actúa,

ii.la estabilidad relacionada con la capacidad para durar en el tiempo y la previsibilidad de lo que pueda pasar en el futuro, $y$

iii.la eficacia y la eficiencia que tiene que ver con alcanzar los objetivos prefijados con el menor costo posible.

La institucionalidad del Estado ha ido generando paulatinamente desconfianza en la sociedad precisamente por su incapacidad de propiciar una gestión de mayor calidad, eficiencia y transparencia en el uso de los recursos públicos, lo cual ha provocado en muchos casos crisis de gobernabilidad.

La pérdida de credibilidad respecto al Estado contemporáneo y al gobierno está estrechamente relacionada con la pérdida de confianza de la ciudadanía hacia la democracia, el sistema político, el sistema legal y las instituciones. El ejercicio del poder ha sido más afín a grupos e individuos vinculados a sectores económicos y 
políticos, que a los intereses nacionales y a la población. Por ello, es de importancia entender la dinámica de la gobernabilidad y el ejercicio del poder y su mediación en el Estado Democrático de Derecho.

\section{¿Democracia para qué?}

Durante las últimas tres décadas del pasado siglo XX, en el mundo en general, América Latina en particular, se ha vivido un proceso de profundas transformaciones una de las cuales ha sido el avance de la democracia como opción de gobierno. En el informe sobre la democracia en América Latina (UNDP, 2004), se plantea que la democracia es una inmensa experiencia humana y que esta ligada a la búsqueda histórica de libertad, justicia y progreso material y espiritual, por ello, la democracia ha sido y continuará siendo, una experiencia inconclusa. (p.33).

En este mismo informe, a partir de los fundamentos teóricos, se argumenta que la democracia i) supone una idea del ser humano y de la ciudadanía; ii) es una forma de organización del poder que implica la existencia y buen funcionamiento del Estado; iii) implica una ciudadanía integral, esto es, el pleno reconocimiento de la ciudadanía política, la ciudadanía civil y la ciudadanía social; y, iv) tiene en el régimen electoral un elemento fundamental, pero no se reduce a las elecciones (UNDP, 2004, p.24).

La democracia reconoce, como condición necesaria, la existencia de un régimen político que se desenvuelve en un Estado y una nación que delimitan una población, un territorio y el poder que se ejerce en su interior. Para Bobbio (1986), la democracia está caracterizada "por un conjunto de reglas (primarias o fundamentales) que establecen quién está autorizado para tomar las decisiones colectivas y bajo qué procedimientos" (p. 14). En ese sentido, la democracia está referido a un conjunto de instituciones y procedimientos que definen las reglas y los canales de acceso a las principales posiciones del Estado, el ejercicio del poder y el proceso de toma de decisiones.

Cuando se habla de "ciudadanía" inmediatamente se asocia con "democracia". Indudablemente podría defenderse algún tipo de ejercicio de ciudadanía fuera de un régimen democrático, pero si se considera la ciudadanía en sus tres componentes básicos (derechos, participación y pertenencia), únicamente en un sistema democrático los ciudadanos pueden desarrollar completamente el conjunto de habilidades, conocimientos y valores que se asocian a esta dimensión política de la persona.

La ciudadanía es la condición mediante la cual un individuo se integra a una sociedad jurídicamente organizada, adquiriendo derechos y obligaciones, es decir, un 
conjunto de derechos y deberes a los cuales el ciudadano o el individuo está sujeto en su relación con la sociedad en que vive. Aunque Bobbio (1986) plantea que el exceso de participación puede tener como efecto de saturación de la política y el aumento de la apatía electoral. Según Bobbio, nada es más peligroso para la democracia que el exceso de democracia (p. 20).

Sin embargo, no todos los sistemas sociales gobernables actúan sobre la base de la democracia. La gobernabilidad democrática se dará sólo cuando la toma de decisiones y la resolución de conflictos entre los actores estratégicos se realice según un sistema de reglas y fórmulas que podamos considerar democráticas.

Los fundamentos de la democracia representativa son una serie de proposiciones relativamente sencillas: i) la idea de nación, como entidad territorial bastante homogénea y diferenciada; ii) la idea de soberanía, como algo ubicado en el Estado, pero que emana del pueblo y, iii) la idea de representación. Estas tres ideas han entrado en crisis progresivamente, debido al desgaste que se viene produciendo entre los ciudadanos en el prestigio de la política, los partidos y las instituciones propias de la democracia representativa. En conexión con este deterioro se observa la falta de eficacia para la selección de líderes, la formulación de programas viables y la administración del poder político.

La crisis de representación y la falta de credibilidad en el sistema político, conlleva en numerosas ocasiones, al surgimiento de liderazgos personalistas que concentran el poder (populismo y nuevas formas de autoritarismo) e impiden el consenso (polarización afectiva) y ciudadanos que confían en ese tipo de líderes y no en las instituciones, o que directamente, se alejan de la vida política activa (desafección democrática y triunfo de movimientos radicales y antisistema).

La inexistencia de liderazgos positivos que proporcionen cohesión tanto en el discurso, como en la política pública que refleje los claros intereses colectivos, no posibilita una confianza ni en la política, ni en el Estado. La desesperanza de la población sobre un Estado y una democracia que no brinda sentido a sus vidas desde la solidaridad, la justicia y la responsabilidad común social es inminente como desafío en la superación del individualismo.

Bobbio (1986) lo plantea con claridad cuando señala que “jamás están ausentes las oligarquías del poder" (p. 21) y resalta a Joseph Schumpeter, a quien considera que captó perfectamente el sentido cuando sostuvo que "la característica de un gobierno democrático no es la ausencia de élites sino la presencia de muchas élites que compiten entre ellas por la conquista del voto popular" (Ibid). En las sociedades actuales es posible y necesario profundizar en la democracia a través de la participación ciudadana y la revitalización del principio del diálogo y la transparencia. 
Lo anterior nos llama a reflexionar sobre la posibilidad de implantar un modelo de gobernanza participativa, un concepto que nos traslada la idea de superación del modelo de gobierno burocrático-jerárquico por un modelo cooperativo más descentralizado que apueste por la complementariedad entre el sector público, el sector privado y las organizaciones, grupos e individuos que conforman la sociedad civil.

Estamos frente a la necesidad de una democracia renovada, una democracia ligada a procesos reales de transformación social, no solo mejorando las reglas como menciona Subirats (2011), sino que consiga garantizar la inclusión de todos en los diversos derechos asociados a la ciudadanía:

i.los derechos civiles, de reconocimiento de la ciudadanía, ii.los derechos políticos de sufragio y representación democrática y, iii.los derechos sociales de salud, educación, vivienda y protección social.

Si hablamos de la búsqueda de la calidad de la democracia, diremos que está vinculada a las nuevas formas de participación lo que hace necesario revisar el concepto de ciudadanía en donde las personas se sientan incluidas en los tres grandes ámbitos como ser el Estado en el reconocimiento de derechos, en la economía al servicio de las personas y en el ámbito social.

Rosanvallon (2009) alude a ello cuando propone renovar el debate sobre la ciudadanía, superando la concepción individualista del mercado, que confunde personalización y singularidad con capacidad de consumo. Debemos, por lo tanto, poner más el énfasis en el ser que en el tener, ir hacia una democracia de mayor calidad que supere las condiciones legales o marcos jurídico, las institucionalidades débiles y una ciudadanía formada, consistente y con una cultura de participación activa en beneficio de la vida plena de todos los ciudadanos.

La prevalencia de intereses individuales y de grupos de elite por sobre los intereses nacionales o de la sociedad en general, es un desafío permanente no solo de la democracia misma, sino de cómo se gestiona el Estado y su institucionalidad. La existencia de los poderes facticos y la competencia entre las élites políticas y económicas han conllevado a crisis y conflictos, que son cada vez más frecuentes. No es que la democracia sea ingenua en el sentido de no estimar que en múltiples ocasiones prevalecen intereses individuales por sobre los intereses comunes de la 
sociedad, pero en la medida que exista expansión de la ciudadanía, gestión eficaz del gobierno y control sobre los recursos públicos se reduce el riesgo de que los temas individuales o de grupos de interés adquieran relevancia, aumentando la posibilidad de ampliar las políticas públicas y los recursos públicos hacia el interés común de la sociedad.

\section{La expansión de la ciudadanía para el desarrollo humano}

La democracia ya no solo depende de que los ciudadanos ejerzan libremente sus derechos políticos, sino que también participen activamente en los diferentes ámbitos y etapas del quehacer público (Vallespín, 2000). Esto nos obliga a repensar no solo en los nuevos roles que habrán de adoptarse en las instituciones básicas para la organización política-administrativa del Estado, sino también a consolidar un conjunto de condiciones sociales que permitan impulsar la construcción de nuevas formas asociativas y de solidaridad social, acentuando la relevancia de la participación ciudadana en la consolidación de las democracias más allá de las representativas.

El concepto de "ciudadanía" proviene etimológicamente del término latino civitas, que significa ciudad, originariamente la unidad política más importante. Con el acontecer del tiempo, la unidad política pasó a ser el Estado y comenzaron a utilizarse las expresiones ciudadano y ciudadanía en el contexto de un Estado, nación o país. La ciudadanía era la condición necesaria para que un individuo fuera reconocido como miembro de una comunidad organizada, por lo que el término confería reconocimiento, aceptación para poder expresar apreciaciones y consideraciones sobre los diferentes aspectos de la vida pública, participar en los debates y decisiones de la comunidad, poder votar en el proceso de elección de los representantes y ser elegido para cargos de representación.

En definitiva, la ciudadanía proporcionaba el acceso a la participación en la política de una comunidad. De ahí que los conceptos de ciudadanía y participación se encuentren estrechamente unidos (García Escribano, 2018, p. 16). Por tanto, el concepto de ciudadanía implica, identidad, pertenencia, participación; lo que explica los modelos de ciudadanía: Liberal-Individualista, Cívica-Republicana y Comunitarista.

La participación ciudadana ha estado debilitada por su ausencia en la toma de decisiones públicas que orienten un desarrollo inclusivo y que favorezcan su gobernanza. La participación ciudadana es un tipo de relación Sociedad/Estado que implica una intención de incidencia de los actores sociales en los asuntos públicos, 
ya sea intercambiando información sobre preferencias, necesidades, propuestas o soluciones sobre los problemas públicos, ya sea ejerciendo un recurso de poder de decisión de manera directa o en conjunto con los actores estatales, o incluso, interviniendo de manera colaborativa en la implementación de las decisiones públicas.

A pesar de que el término ciudadanía se conoce y se aplica desde la Grecia clásica, no es hasta bien entrado el siglo XX cuando se trata de conceptualizar de forma sistemática. El responsable fue T. H. Marshall (1950), que hizo una contribución trascendental a las teorías de la ciudadanía al incluir los derechos sociales en la definición de la ciudadanía moderna. Marshall (1997) escribió que la ciudadanía es "un estatus que se otorga a los que son miembros de pleno derecho de una comunidad. Todos los que poseen ese estatus son iguales en lo que se refiere a los derechos y deberes que implica" (p. 312).

Desde esta perspectiva, todas las personas de una comunidad política determinada son iguales, son ciudadanos, porque disfrutan en la misma medida de un conjunto de derechos civiles, políticos y sociales. Marshall propuso una secuencia de la ampliación de los derechos de la ciudadanía: los derechos civiles (concedidos en el siglo XVIII), los derechos políticos (siglo XIX) y los derechos sociales y económicos (siglo XX), que los individuos han obtenido al ser reconocidos por el Estado, ente que ha regulado legalmente estos derechos.

Como señala Horrach (2009), el "modelo liberal de ciudadanía, hegemónico durante décadas, se caracteriza por defender la libertad, entendiéndose como la no interferencia del Estado con respecto a la voluntad soberana del individuo. Desde su perspectiva, la finalidad del Estado es puramente instrumental. Por otro lado, el modelo de ciudadanía republicana ha ido adquiriendo más relevancia en la segunda mitad del siglo XX. De todas formas, aunque se plantee como una alternativa al citado liberalismo, no pocos autores consideran que es inevitable un acuerdo entre una y otra opción, un modelo mixto. Enlaza con el republicanismo clásico, aunque ha sabido adaptar en muchas ocasiones sus tesis a la modernidad. Sus autores más representativos son Habermas, Hannah Arendt, J.G.A. Pocock, Quentin Skinner o Philip Pettit” (p. 15).

Actualmente existe la falta de apropiación de los espacios locales destinados al debate y la participación por parte de los ciudadanos, los alcaldes municipales que fueron electos no logran tener una relación de representación y confianza con el ciudadano, y más bien se basa en una relación particularista y clientelar que hace que la figura del alcalde deje de ser un representante de los habitantes de la localidad, y pase a ser un facilitador de las necesidades de la población local. Entonces se pudiera pensar cómo llegar a tener un espacio más orientado a la construcción de 
una ciudadanía activa y participativa en los asuntos públicos y menos un espacio en donde se canalizan demandas que pueden ser atendidas o no.

Hablar de ciudadanía y participación ciudadana está relacionado con el ciudadano, con capacidad para hacerle frente a procesos de transformación tanto nacional, como regional y local. Estamos hablando de actores que posicionan propuestas alternativas para que la humanidad pueda progresar hacia los ideales de paz, libertad y justicia social.

Ante el bajo nivel de participación ciudadana en el logro de los objetivos del desarrollo humano en la gestión pública en niveles nacionales, regionales y locales, así como la necesidad de más transparencia y vigilancia ciudadana de la inversión pública y que en la mayoría de los casos en los municipios la participación se queda meramente en la parte electoral y en la ejecución, administración y control de proyectos, la participación ciudadana sólo se hace a nivel de consultas en la fase de elaboración de los planes de desarrollo municipal, pero no en la ejecución de los proyectos y menos en la toma de decisiones públicas que orienten un desarrollo inclusivo en los municipios y fortalezcan la gobernanza.

En el proceso de la pérdida de credibilidad y de confianza en los sistemas políticos y en las instituciones públicas, la ciudadanía aparece como una preocupación en medio de dicho proceso que deslegitima todo lo público y pérdida de derechos que hace que la población demande más justicia pero que la individualización de las acciones en una sociedad que va perdiendo el sentido de pertenencia repercute en sus derechos sociales y políticos.

La participación ciudadana es un tipo de relación Sociedad/Estado que implica una intención de incidencia de los actores sociales en los asuntos públicos, ya sea intercambiando información sobre preferencias, necesidades, propuestas o soluciones sobre los problemas públicos, ya sea ejerciendo un recurso de poder de decisión de manera directa o en conjunto con los actores estatales, o incluso, interviniendo de manera colaborativa en la implementación de las decisiones públicas.

De acuerdo con Espinosa (2009, pp.97-98), en su artículo "La participación ciudadana como una relación socio-estatal acotada por la concepción de democracia y ciudadanía", resalta que la ciudadanía es una construcción social, ya que nos remite a un proceso que se encuentra fuertemente vinculado con el ejercicio y/o desarrollo de procesos ubicados en tres dimensiones:

i)la civil, dimensión en que el objeto de la acción es la defensa de los derechos de igualdad ante la ley, libertad de la persona, libertad de expresión, libertad 
de información, libertad de conciencia, de propiedad y de la libertad de suscribir contratados;

ii)la política, dimensión en que el objeto de la acción está relacionado con el derecho de asociación y con el derecho a participar en el poder político, tanto en forma directa, por medio de la gestión gubernamental, como de manera indirecta, a través del sufragio;

iii)la social, dimensión en que el objeto de la acción nos remite al conjunto de derechos de bienestar (mínimos) y obligaciones sociales que permiten a todos los miembros participar en forma equitativa de los niveles básicos de la vida de su comunidad.

Conforme a lo anterior, Espinosa (2009) concluye que hay tres elementos de la participación ciudadana que resultan clave:

i) la ciudadanía, organizada o a título individual, debe asumir un rol activo y relevante; ii) la participación debe producirse sobre cuestiones de carácter público, entendiendo lo público como lo colectivo, más allá del marco institucional;

iii) a través de su participación, las personas han de incidir en la resolución de problemas de naturaleza colectiva y, por tanto, también en los resultados, ya sea aportando elementos de diagnóstico, planteando propuestas, tomando decisiones o implementando soluciones.

Como se puede observar, este conjunto de derechos, mediante los que se describe la ciudadanía, corresponden a un modelo ideal de relaciones sociopolíticas que acotan los espacios de participación ciudadana.

Es importante resaltar que Espinosa (2009) considera importante distinguir entre la participación ciudadana de la participación política porque "el conjunto de actos y relaciones supuestas en el desarrollo de la primera no están enfocados (exclusiva, ni fundamentalmente) a influir en las decisiones y la estructura de poder del sistema político. Es decir, aun cuando con el despliegue de estas prácticas ciudadanas se busca incidir en la toma de decisiones que constituyen el orden de la política y de las políticas, se diferencian sustancialmente de las actividades políticas porque el conjunto de acciones, desplegadas desde este ámbito ciudadano, no pretende ser ni constituirse en poder político, ni busca rivalizar con éste" (pp. 75-76).

Ahora bien, las prácticas relacionadas con estos procesos participativos no sólo están ceñidas a dichos contornos, sino que también apuntan dinámicas y maneras específicas de entender su sentido u orientación específica. Por ejemplo, las prácticas o estrategias ciudadanas pueden tener una dinámica autónoma (emerger "exclusivamente" de los movimientos sociales y ser acciones reivindicativas de los 
derechos sociales, políticos y civiles) o caracterizarse por una dinámica dependiente, esto es, corresponder más con un despliegue de estrategias paternalistas y clientelares de la acción gubernamental, que tienen por objeto la procuración de una cierta legitimidad política, así como el control del orden y el poder político, antes que el fortalecimiento y construcción de una ciudadanía integral (Bayón, Bryan y Saraví, 1998, p. 84).

\section{Los desafíos contemporáneos del Estado, la democracia y la ciudadanía}

La transformación del Estado debería ser entendida como un proceso inminente y permanente entendiendo que las sociedades y sus relaciones son dinámicas y que las realidades sociales, económicas, culturales y ambientales son cambiantes. Esto se debe, entre otros factores, a que, bajo el panorama actual de la gobernanza, en el nuevo orden global, el Estado no debe y no puede poseer el monopolio del conocimiento, la experiencia y los recursos necesarios para resolver por sí sólo los problemas y obtener oportunidades eficientemente.

El Estado continúa siendo protagonista de la escena, teniendo, entre otros, los siguientes desafíos que afrontar:

i) posibilitar un desarrollo económico sostenible e incluyente;

ii) superar la pobreza y la desigualdad;

iii) garantizar el acceso a la salud y educación pública con calidad;

iv) posibilitar una sociedad más justa y que permeabilice las demandas de los ciudadanos;

v) facilitar la superación de la desafección y la apatía política;

vi) potenciar los efectos positivos y neutralizar los efectos negativos de la globalización; vii) proteger los derechos humanos, viabilizando la construcción de una sociedad multicultural y de integración.

Con relación a la democracia, Bitar (2019) plantea que América Latina debe encarar cuatro mayores desafíos, los cuales están relacionados con desafíos políticos institucionales, desafíos económicos, desafíos de cohesión social y desafíos culturales. Entre ellos, Bitar señala una serie de aspectos como la crisis de representatividad ya que desafía la gobernabilidad democrática. Asimismo, apunta a la realización de reformas a fin de encauzar institucionalmente las demandas sociales por mayor igualdad y participación, adoptando políticas públicas relacionadas con nuevas formas de participación y consulta, establecimiento de una institucionalidad amplia e incluyente que promueva el dialogo social y reformas del Estado que permita gobernar sociedades complejas. 
Conforme a lo anterior, Prats asevera que "la reforma del Estado tiende hoy justamente en América Latina a plantearse en términos de gobernabilidad". Lo importante a destacar en Prats es que considera que sin gobernabilidad no puede haber desarrollo y que la gobernabilidad depende fundamentalmente de la estructura institucional formal e informal existente y de las capacidades o competencias de los actores de la gobernación (p. 474).

El desarrollo global debe fundamentarse en un desarrollo sostenible, centrado en las personas, equitativo y con protección social. Reconocemos que el crecimiento económico y la creación de riqueza debería ser inclusiva, con acciones de políticas de desarrollo centrado en la reducción de la pobreza, la protección ambiental, generación de empleos dignos y calidad de vida para todos. De acuerdo con Salama (2014), el desarrollo sostenible "impone un cambio en las relaciones del ser humano con la naturaleza y en las relaciones entre los individuos en pos de una sociedad más democrática e inclusiva “(p. 90).

Para Oriol Prats (2003), al existir gobernabilidad asociada con mayores niveles de democracia y bienestar, serán las fórmulas democráticas las que mejor canalicen las necesidades y las demandas ciudadanas y será en términos de bienestar entendido como desarrollo humano que se juzgue la bondad de las políticas públicas. (p. 250).

En relación con el gobierno abierto, es "aquel que entabla una constante conversación con los ciudadanos con el fin de escuchar lo que ellos dicen y solicitan, que toma decisiones basadas en sus necesidades y teniendo en cuenta sus preferencias, que facilita la colaboración de los ciudadanos y funcionarios en el desarrollo de los servicios que presta, y que comunica todo lo que decide y hace de forma abierta y transparente" (Calderón y Lorenzo, 2010, p. 11). El gobierno abierto es una oportunidad de poder incidir a través de este hacia una participación ciudadana más activa que pueda generar los cambios que la población demanda.

Cada vez más se observa a los ciudadanos menos identificados con el ideal democrático, por lo que la renovación de la democracia debe ir acompañada de una participación formada y consiente para evitar que en vez de fortalecer una verdadera democracia deriven a gobiernos autocráticos en donde la concentración del poder conlleva a una institucionalidad democrática débil que erosiona las libertades civiles. Siguiendo a Pastor (2008), "la participación ciudadana no se plantea como alternativa a las instituciones representativas, pero sí como complemento que facilita los procesos decisivos, haciéndolos más transparentes, dándoles más legitimación, incluyendo más puntos de vista e información y restituyendo la confianza de los ciudadanos en sus instituciones" (p. 140). 
Por ello, la UNESCO (2018), plantea una educación para una ciudadanía mundial, lo cual implica cuatro aspectos a considerar: i) una educación para los derechos humanos, ii) una educación para la paz, iii) una educación para el desarrollo sostenible y, iv) una educación para el entendimiento internacional. Formar y educar para una interdependencia política, social y cultural entre las personas, requiere articular lo local, lo nacional y lo global para la construcción de conocimientos y sentidos comunes que nos permitan convivir en paz y bajo una justicia social (p. 8).

No hay duda de que se requiere un Estado que ampare los derechos humanos. "Colocar los derechos humanos en la agenda pública y política puede abrir el camino a la construcción de una verdadera democracia" (ONU, 2016, p. 355). Hay que considerar que los derechos humanos en la región latinoamericana han sido muy importantes en procesos democratizadores, en los cuales han servido para mostrar las diferencias que existen entre regímenes y los derechos humanos como articulador de la agenda política y cohesionador de las diversas tendencias de la sociedad civil hacia la búsqueda de la democracia.

\section{CONCLUSIÓN}

Para concluir, consideramos que la inclusión equitativa puede conducir a una democracia plena. Por ello, se debe buscar un equilibrio entre lo económico, social y medioambiental a fin de superar los procesos de desarrollo geográfico desigual, la pobreza extrema, la precarización del empleo, las migraciones forzadas, el desarraigo, el racismo, la xenofobia, la desigualdad y la violencia de género, entre otros, desarrollando nuestra capacidad para enfrentar los cambios y construyendo una sociedad de ciudadanía, con reconocimiento de igualdad de derechos, teniendo como premisa fundamental, la vida misma.

\section{BIBLIOGRAFÍA}

-BAUMAN, Zygmunt (2008). La Gran Separación, segunda fase. En La Sociedad sitiada, Buenos Aires: FCE. (pp. 71-112).

-BECK, Ulrich (1998). La sociedad del riesgo: Hacia una nueva modernidad, Paidós -BECK, Ulrich (2017). Las generaciones del riesgo global: unidas en la decadencia, En La metamorfosis del mundo, Barcelona: Paidós. (pp. 169-178).

-BITAR, Sergio (2019). Los desafíos de la democracia en América Latina. IDEA. En: https://www.idea.int/es/news-media/news/es/los-desaf\%C3\%ADos-la-democraciaen-am $\% \mathrm{C} 3 \%$ A9rica-latina 
-BOBBIO, Nolberto (1986). El futuro de la democracia. México: FCE.

-BOBBIO Norberto; MATTEUCCI, Nicolla y PASQUINO, Gianfranco (2002). Diccionario de Política, México: Siglo XXI.

-CALDERÓN, César. y LORENZO, Sebastián (coords). (2010). Open Government: Gobierno abierto. Jaén: Algón editores.

-CAMOU, Antonio (2013). Gobernabilidad y Democracia. México: Instituto Federal Electoral.

-DOMINGUEZ AVILA, Carlos Federico (2009). Honduras: vicisitudes en la construcción de una democracia de ciudadanía, Memorias. Revista Digital de Historia y Arqueología desde el Caribe, 11, 220-232.

-ESPINOSA, Mario (2009). La participación ciudadana como una relación socioestatal acotada por la concepción de democracia y ciudadanía. Revista Andamios 5, (10), 71-109.

-GARCÍA ESCRIBANO, Juan José (2018). Sociedades actuales: reconsideración de la ciudadanía y la participación. En Nelly Caro Luján y José A. Rodríguez Alonso (Coord.): Ciudadanía y participación. Una mirada desde lo local, Zinacantepec: Colegio Mexiquense, A. C. (pp. 15-41).

-HERRERA GÓMEZ, Manuel y SORIANO MIRÁS, Rosa María (2005), De las versiones modernas de la ciudadanía a la ciudadanía de las autonomías sociales de la postmodernidad, Revista Española de Investigaciones Sociológicas, 112, 43-74.

-HORRACH, Juan Antonio (2009). Sobre el concepto de ciudadanía: historia y modelos. Factótum, 6, 1-22.

-KOOIMAN, J., Findings (1993). Speculations and Recomendations, en: Kooiman (ed.), Modem Governance. London: Sage Publications.

-MARSHALL, Thomas Humphrey (1997). Ciudadanía y clase social. Revista Española de Investigaciones Sociológicas, 79, 297-344.

- UNDP (2004). La democracia en América Latina. Hacia una democracia de ciudadanas y ciudadanos. Buenos Aires: Alfaguara S.A.

-ONU (2016). Agenda 2030 y los Objetivos de Desarrollo Sostenible: Una oportunidad para América Latina y el Caribe. Santiago.

-ORIOL PRATS, Joan (2003). El concepto y el análisis de la gobernabilidad. Instituciones y Desarrollo, 14-15, 239-269.

-PASTOR SELLER, Enrique. (2009). Participación ciudadana y gestión de las políticas sociales municipales. Murcia: Editum

-PRATS, Joan (1996). Gobernabilidad democrática en la América Latina finisecular. Instituciones, gobiernos y liderazgo, Estudios Internacionales, 29 (116), 447-492.

-SALAMA, Pierre (2014). ¿Es posible otro desarrollo en los países emergentes? Revista Nueva Sociedad, 250.

-SANTOS, Boaventura de Sousa (2011). Reinventar la democracia, en El milenio huérfano. Ensayos para una nueva cultura política, Bogotá: Trotta. 
-SUBIRATS, Joan (2011). Nueva ciudadanía y la dinámica de lo común. En Otra sociedad ¿otra política?: De «no nos representan» a la democracia de lo común, Barcelona: Icaria Asaco (pp. 81-97).

-ROSANVALLON, Pierre (2009). La democracia y sus condiciones. Cuadernos del Cendes, 26(71), 149-160.

-UNESCO (2018). Educación para la ciudadanía mundial. Santiago de Chile.

-VALLESPÍN, Fernando (2000). El futuro de la política. Madrid: Taurus.

-ZICCARDI, Alicia (2004). Participación ciudadana y políticas sociales del ámbito local. México: Universidad Nacional Autónoma de México. Instituto de Investigaciones Sociales.

\section{CÍRCULO TEMÁTICO: ESTADO, CIUDADANÍA, GOBERNABILIDAD, DESCENTRALIZACIÓN Y DERECHOS HUMANOS}

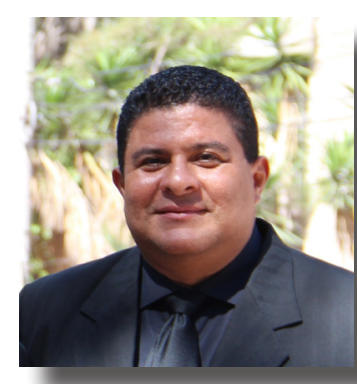

Juan Carlos Benitez

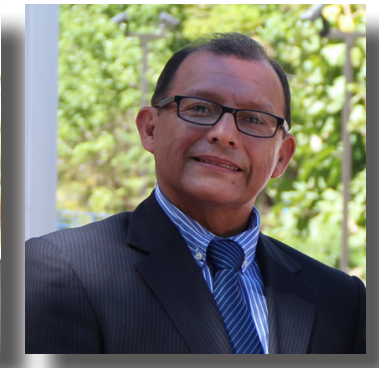

Orlando Galeano

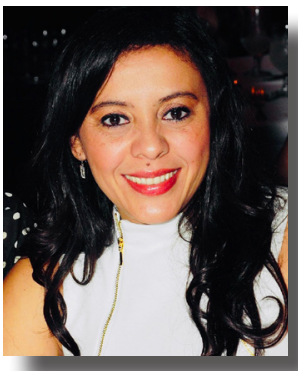

Jenny S. Suazo Leonardo Euceda 